\title{
Experimentally testable state-independent quantum contextuality
}

\author{
Adán Cabello1* \\ ${ }^{1}$ Departamento de Física Aplicada II, Universidad de Sevilla, E-41012 Sevilla, Spain
}

(Dated: October 24, 2018)

\begin{abstract}
We show that there are Bell-type inequalities for noncontextual theories that are violated by any quantum state. One of these inequalities between the correlations of compatible measurements is particularly suitable for testing this state-independent violation in an experiment.

PACS numbers: 03.65.Ta, 03.65.Ud, 42.50.Xa, 03.75.Dg
\end{abstract}

Because of the lack of spacelike separation between one observer's choice and the other observer's outcome, the immense majority of the experimental violations of Bell inequalities does not prove quantum nonlocality, but just quantum contextuality. Bell inequalities can only be violated by entangled states. However, in principle, Belltype inequalities for noncontextual theories might be violated by any quantum state.

Bell's theorem states that no theory of local hidden variables can reproduce quantum mechanics $(\mathrm{QM})[1]$. It is proven either by the violation of a Bell inequality [1, 2] or by a logical contradiction between the LHV predictions and those of QM [3]. Bell inequalities have some advantages. They are independent of QM, testable in experiments, and have applications in communication complexity [4], entanglement detection [5], security of key distribution [6], and quantum state discrimination [7]. Any proof of Bell's theorem is state-dependent: it is valid for some states but not for others.

Local hidden variable theories are a special type of noncontextual hidden variable (NCHV) theories, defined as those where the expectation value of an observable $A$ is the same whether $A$ is measured with a compatible observable $B$, or with a compatible observable $C$, even though $B$ and $C$ are incompatible. The Kochen-Specker (KS) theorem states that no NCHV theory can reproduce QM [8, 9, 10]. The KS theorem is proven by a logical contradiction [10, 11, 12, 13, 14, 15, 16]. These proofs apply to systems described by Hilbert spaces of dimension $d \geq 3$ and are state-independent (i.e., valid for any state). Quantum contextuality is related to quantum error correction [17], random access codes [18], quantum key distribution [19], one-location quantum games [20], and entanglement detection between internal degrees of freedom.

The differences between the proofs of Bell's and the KS theorems lead to the question of what is the connection between them. It has been shown [21, 22, 23] that any proof of the KS theorem can be converted into a proof of impossibility of "elements of reality" 24]. Some proofs of the KS theorem can be converted into logical proofs of Bell's theorem [12, 14] which can be translated into Bell inequalities [25].

The differences between the proofs are also in the heart of the controversy on whether experimental tests of the
KS theorem make sense [26] or are even possible if the finite precision of measurements is taken into account 27, 28, 29, 30, 31, 32, 33, 34, 35, 36.

As a result of these debates, two types of inequalities to test quantum contextuality have been proposed. On one hand, there are "KS inequalities" [33, 34], which are based on the assumption of contextuality and on some QM predictions, and therefore are not independent of QM. On the other hand, there are inequalities that are based only on the assumption of noncontextuality, in the same way that the Bell inequalities are based only on the assumption of locality. These inequalities are independent of QM and testable in experiments. There are recent proposals for testing inequalities of this type in different physical systems 37, 38, 39]. However, the fact that all these inequalities are state-dependent, while the proofs of the KS theorem are state-independent, has been recently described as "a drawback" [38]. A natural question is the following: Given a physical system described in QM by a Hilbert space of dimension $d$, is it possible to derive experimentally testable inequalities using only the assumption of noncontextuality, such that any quantum state violates them?

We describe the first inequalities of this type. Each of them is valid for a value of $d$, and all of them share a curious property. Then, we will discuss how these inequalities may be tested, and whether the state-independent violation predicted by QM can be observed in actual experiments.

First inequality.-Suppose that $A_{i j}$ is an observable with two possible results: -1 or +1 , and two observables $A_{i j}$ and $A_{k l}$ are compatible if they share a subindex (i.e., $i=k$, or $i=l$, or $j=k$, or $j=l$ ). When we prepare an ensemble of systems and measure 4 compatible observables $A_{i j}, A_{i k}, A_{i l}$, and $A_{i m}$ in each system, $\left\langle A_{i j} A_{i k} A_{i l} A_{i m}\right\rangle$ denotes the average of the products of their results. In any theory of NCHV in which the observables $A_{i j}$ have definite results, the following inequality must be satisfied:

$$
\begin{aligned}
& -\left\langle A_{12} A_{16} A_{17} A_{18}\right\rangle-\left\langle A_{12} A_{23} A_{28} A_{29}\right\rangle-\left\langle A_{23} A_{34} A_{37} A_{39}\right\rangle \\
& -\left\langle A_{34} A_{45} A_{47} A_{48}\right\rangle-\left\langle A_{45} A_{56} A_{58} A_{59}\right\rangle-\left\langle A_{16} A_{56} A_{67} A_{69}\right\rangle \\
& -\left\langle A_{17} A_{37} A_{47} A_{67}\right\rangle-\left\langle A_{18} A_{28} A_{48} A_{58}\right\rangle \\
& -\left\langle A_{29} A_{39} A_{59} A_{69}\right\rangle \leq 7 .
\end{aligned}
$$




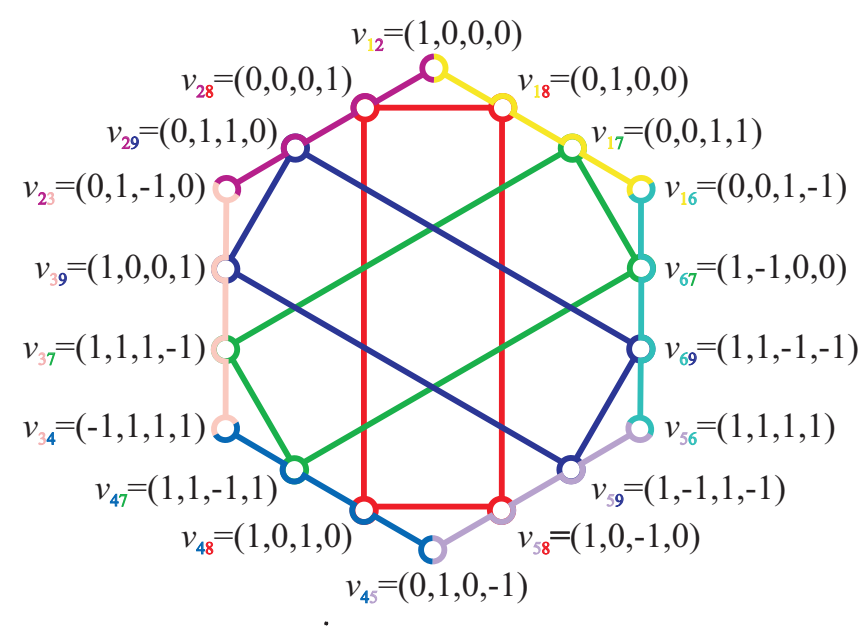

FIG. 1: Each dot represents a unit-vector $v_{i j}$. Each of the 6 sides of the regular hexagon and each of the 3 rectangles contains only orthogonal vectors. Note that, for clarity's sake, most labels have no unit length.

This can be proven as follows. We define $\alpha=$ $-A_{12} A_{16} A_{17} A_{18}-\ldots-A_{29} A_{39} A_{59} A_{69}$. If we generate all the $2^{18}$ possible values of $\alpha$, we will find that $\alpha=7$ is the maximum. Therefore, if we can measure $\alpha$ on different systems, the average satisfies $\langle\alpha\rangle \leq 7$. We cannot measure $\alpha$ on a single system, because $\alpha$ contains incompatible observables. However, since we are assuming that each $A_{i j}$ would give the same result in any context, we can measure subsets of compatible observables on different subensembles prepared in the same state, and then inequality (1) is valid for the averages over each subsensemble. This derivation is similar to a standard derivation of a Bell inequality. The only difference is that in a Bell inequality we assume that the result of a measurement of $A_{12}$ is independent of spacelike separated measurements, while here we assume that it is independent of compatible measurements.

Now consider a physical system described by a Hilbert space of dimension $d=4$ (e.g., two qubits or a single spin-3/2 particle), and the observables represented by the operators

$$
A_{i j}=2\left|v_{i j}\right\rangle\left\langle v_{i j}\right|-\mathbb{1},
$$

where $v_{i j}$ is a unit vector and $\mathbb{1}$ denotes the identity. Each observable $A_{i j}$ has two possible results: -1 or +1 . If $v_{i j}$ is orthogonal to $v_{i k}$, then $A_{i j}$ and $A_{i k}$ are compatible. Therefore, 4 orthogonal vectors define 4 compatible observables. 18 vectors $v_{i j}$ with the the orthogonality relations assumed in inequality (1) are presented in Fig. 1.

Let us prove that, for $d=4, \mathrm{QM}$ violates (11) for any state. According to QM, if one measures on the same system 4 compatible observables $A_{i j}$ corresponding to 4 orthogonal vectors $v_{i j}$, the product of their 4 results will always be -1 , because $A_{i j} A_{i k} A_{i l} A_{i m}=-\mathbb{1}$. Therefore, using the vectors of Fig. 1] QM predicts that the experimental value of the left-hand side of inequality (1) must be 9 in any state, which is clearly beyond the bound for any description based on noncontextual hidden variables.

Relation to previous results. - The 18 vectors in Fig. 1 have also be used for a proof of the KS theorem [16], in which it is assumed: (I) that the observables represented by the projectors $\left|v_{i j}\right\rangle\left\langle v_{i j}\right|$ have noncontextual results 0 or 1 , and (II) that the results of 4 compatible projectors are one 1 and 3 zeroes (a QM prediction). A simple parity argument proves that it is impossible to assign values satisfying both (I) and (II): There are 9 (an odd number) complete sets of projectors, while each projector appears in two (an even number) of them [16]. Here we have used the 18 vectors of Fig. 1 for a different purpose: (1) is an experimentally testable inequality, not a proof by contradiction.

Other interesting relation of inequality (1) to previous results is the following. In Ref. 38 there is a statedependent inequality for testing $\mathrm{NCHV}$ theories in systems of $d=3$ (e.g., spin-1 particles). Inequality (5) in Ref. [38] can be expressed as

$$
\begin{array}{r}
-\left\langle A_{12} A_{18}\right\rangle-\left\langle A_{12} A_{23}\right\rangle-\left\langle A_{23} A_{34}\right\rangle-\left\langle A_{34} A_{48}\right\rangle \\
-\left\langle A_{18} A_{48}\right\rangle \leq 3 .
\end{array}
$$

Using the observables $A_{i j}$ defined before, it is easy to see that, for the state $(\cos 0.3, \sin 0.3) \otimes(\cos 0.7,-\sin 0.7)$, the left-hand side of (3) is 3.6. Therefore, this two-qubit state violates inequality (3). However, other states, e.g. the state $(1,0) \otimes(1,0)$, do not violate it. The interesting observation is that inequality (3) is a particular case of inequality (1): It can be obtained from (1) by replacing 13 out of the 18 observables $A_{i j}$ with identities. While (3) is a state-dependent inequality for systems of $d=$ 3 and $d=4$ [38], (11) is a state-independent inequality for systems of $d=4$. Note that (11) contains several inequalities like (3).

Second inequality. - Suppose that $P_{i j}$, with $i \in\{1,2,3\}$ and $j \in\{4,5,6\}$, is an observable with two possible results: -1 or +1 , and two observables $P_{i j}$ and $P_{k l}$ are compatible if they share a subindex. Using the method described before, it can be easily proved that any NCHV theory in which the observables $P_{i j}$ have definite results satisfies the following inequality:

$$
\begin{array}{r}
\left\langle P_{14} P_{15} P_{16}\right\rangle+\left\langle P_{24} P_{25} P_{26}\right\rangle+\left\langle P_{34} P_{35} P_{36}\right\rangle+\left\langle P_{14} P_{24} P_{34}\right\rangle \\
+\left\langle P_{15} P_{25} P_{35}\right\rangle-\left\langle P_{16} P_{26} P_{36}\right\rangle \leq 4 .(4)
\end{array}
$$

However, if we consider a two-qubit system and choose the following observables:

$$
\begin{aligned}
& P_{14}=Z_{1}, \quad P_{15}=Z_{2}, \quad P_{16}=Z_{1} \otimes Z_{2}, \\
& P_{24}=X_{2}, \quad P_{25}=X_{1}, \quad P_{26}=X_{1} \otimes X_{2}, \\
& P_{34}=Z_{1} \otimes X_{2}, \quad P_{35}=X_{1} \otimes Z_{2}, \quad P_{36}=Y_{1} \otimes Y_{2},
\end{aligned}
$$


where, e.g., $Z_{1}$ denotes $\sigma_{z}^{(1)}$, the Pauli matrix $Z$ of qubit 1, then, according to QM, the left-hand side of (4) must be 6 , since $P_{14} P_{15} P_{16}=P_{24} P_{25} P_{26}=P_{34} P_{35} P_{36}=$ $P_{14} P_{24} P_{34}=P_{15} P_{25} P_{35}=-P_{16} P_{26} P_{36}=\mathbb{1}$. Therefore, $\mathrm{QM}$ violates inequality (4) for any two-qubit state.

Relation to previous results. - The observables (5a)(5c) have been used in the proof of the KS theorem for two-qubit systems proposed by Peres and Mermin [11, 12, 14]. This proof is also based on a parity argument. Again, a testable inequality is connected to a KS proof by contradiction based on a parity argument.

Other interesting connections of inequality (44) to some recent results are the following. In Ref. [37 there is a state-dependent inequality for testing quantum contextuality in two-qubit systems. Inequality (4) in Ref. 37. can be expressed as

$$
\begin{array}{r}
-\left\langle P_{14} P_{15}\right\rangle-\left\langle P_{24} P_{25}\right\rangle-\left\langle P_{34} P_{35}\right\rangle+\left\langle P_{14} P_{24} P_{34}\right\rangle \\
+\left\langle P_{15} P_{25} P_{35}\right\rangle \leq 3 .
\end{array}
$$

According to QM, for the singlet state, the left-hand side of inequality (6) is 5 [37]. What is interesting is that inequality (6) is a particular case of inequality (4), when $P_{16}, P_{26}$, and $P_{36}$ are replaced with $-\mathbb{1}$.

Moreover, the recent proposal for testing quantum contextuality in two-qubit systems in Ref. [39] can be reformulated as the following inequality:

$$
\begin{array}{r}
\left\langle P_{14} P_{15} P_{16}\right\rangle+\left\langle P_{24} P_{25} P_{26}\right\rangle+\left\langle P_{34} P_{35}\right\rangle+\left\langle P_{14} P_{24} P_{34}\right\rangle \\
+\left\langle P_{15} P_{25} P_{35}\right\rangle-\left\langle P_{16} P_{26}\right\rangle \leq 4,(7)
\end{array}
$$

which is maximally violated by the product state $\mid \sigma_{y}^{(1)}=$ $+1\rangle \otimes\left|\sigma_{y}^{(2)}=+1\right\rangle$. Inequality (7) is a particular case of inequality (4) when $P_{36}$ is replaced with $\mathbb{1}$. The fact that a product state violates inequality (7) is not surprising, since any state violates inequality (4).

Finally, if $P_{15}=P_{25}=P_{34}=P_{35}=P_{36}=\mathbb{1}$, then inequality (4) becomes

$$
\left\langle P_{14} P_{16}\right\rangle+\left\langle P_{24} P_{26}\right\rangle+\left\langle P_{14} P_{24}\right\rangle-\left\langle P_{16} P_{26}\right\rangle \leq 2,
$$

which has the same structure of the Clauser-HorneShimony-Holt Bell inequality [2].

Third inequality. - Suppose that the $4+2 n$ observables $\mathcal{A}_{1}, \ldots, \mathcal{A}_{4}, \mathcal{B}_{1}, \ldots, \mathcal{B}_{n}, \mathcal{C}_{1}, \ldots, \mathcal{C}_{n}$, with $n$ (odd) $\geq$ 3 , have only two possible results: -1 or +1 . Assuming that each of the following averages contains only compatible observables, using the method described before, it can be easily seen that any NCHV theory satisfies the following inequality:

$$
\begin{aligned}
\left\langle\mathcal{A}_{1} \mathcal{B}_{1} \mathcal{B}_{2} \prod_{i=3}^{n} \mathcal{B}_{i}\right\rangle & +\left\langle\mathcal{A}_{2} \mathcal{B}_{1} \mathcal{C}_{2} \prod_{i=3}^{n} \mathcal{C}_{i}\right\rangle \\
+\left\langle\mathcal{A}_{3} \mathcal{C}_{1} \mathcal{B}_{2} \prod_{i=3}^{n} \mathcal{C}_{i}\right\rangle & +\left\langle\mathcal{A}_{4} \mathcal{C}_{1} \mathcal{C}_{2} \prod_{i=3}^{n} \mathcal{B}_{i}\right\rangle \\
& -\left\langle\mathcal{A}_{1} \mathcal{A}_{2} \mathcal{A}_{3} \mathcal{A}_{4}\right\rangle \leq 3 .
\end{aligned}
$$

However, if we consider an $n$-qubit system, with $n$ (odd) $\geq 3$, and choose the following observables:

$$
\begin{aligned}
& \mathcal{A}_{1}=Z_{1} \otimes Z_{2} \otimes Z_{3} \otimes \ldots \otimes Z_{n}, \\
& \mathcal{A}_{2}=Z_{1} \otimes X_{2} \otimes X_{3} \otimes \ldots \otimes X_{n}, \\
& \mathcal{A}_{3}=X_{1} \otimes Z_{2} \otimes X_{3} \otimes \ldots \otimes X_{n}, \\
& \mathcal{A}_{4}=X_{1} \otimes X_{2} \otimes Z_{3} \otimes \ldots \otimes Z_{n}, \\
& \mathcal{B}_{i}=Z_{i}, \\
& \mathcal{C}_{i}=X_{i},
\end{aligned}
$$

then, according to QM, the left-hand side of inequality (9) must be 5, since, $\mathcal{A}_{1} \mathcal{B}_{1} \mathcal{B}_{2} \prod_{i=3}^{n} \mathcal{B}_{i}=$ $\mathcal{A}_{2} \mathcal{B}_{1} \mathcal{C}_{2} \prod_{i=3}^{n} \mathcal{C}_{i}=\mathcal{A}_{3} \mathcal{C}_{1} \mathcal{B}_{2} \prod_{i=3}^{n} \mathcal{C}_{i}=\mathcal{A}_{4} \mathcal{C}_{1} \mathcal{C}_{2} \prod_{i=3}^{n} \mathcal{B}_{i}=$ $-\mathcal{A}_{1} \mathcal{A}_{2} \mathcal{A}_{3} \mathcal{A}_{4}=\mathbb{1}$. Therefore, QM violates inequality (9) for any $n$-qubit state with $n$ (odd) $\geq 3$.

Relation to previous results. - For $n=3$, the observables (10a) - (10f) have been used in a proof of the KS theorem for 3-qubit systems proposed by Mermin [12, 14]. Again, Mermin's KS proof is a proof by contradiction based on a parity argument.

On the other hand, taking $\mathcal{A}_{1}=\mathcal{A}_{2}=\mathcal{A}_{3}=-\mathcal{A}_{4}=$ $-\mathbb{1}$, inequality (9) becomes

$$
\begin{array}{r}
\left\langle\mathcal{B}_{1} \mathcal{B}_{2} \prod_{i=3}^{n} \mathcal{B}_{i}\right\rangle+\left\langle\mathcal{B}_{1} \mathcal{C}_{2} \prod_{i=3}^{n} \mathcal{C}_{i}\right\rangle+\left\langle\mathcal{C}_{1} \mathcal{B}_{2} \prod_{i=3}^{n} \mathcal{C}_{i}\right\rangle \\
-\left\langle\mathcal{C}_{1} \mathcal{C}_{2} \prod_{i=3}^{n} \mathcal{B}_{i}\right\rangle \leq 2
\end{array}
$$

What is interesting is that inequality (11) is not only a state-dependent inequality to test quantum contextuality, but also a Bell inequality. Indeed, for $n=3$, inequality (11) is the 3-party Bell inequality discovered by Mermin [25]. For higher values of $n$, inequality (11) is not the Mermin inequality [25], but a new Bell inequality.

Experimental violation.-Observing the stateindependent violation predicted by QM in an actual experiment is a major challenge for the near future. Inequality (4) seems particularly suitable for that purpose, since most of the requirements for the experiment have been addressed, at least in the case where the physical system is a two-qubit system consisting of the spatial and spin components of a single neutron 37]. Other possibility is using the polarization and path degrees of freedom of a single photon [36]. Using the polarization of two photons, as proposed by 39], requires further investigation in order to fulfill all the requirements of the experiment.

To test inequality (4), one has to prepare a specific twoqubit quantum state (e.g., a maximally entangled state), measure, e.g., $P_{14}, P_{15}$, and $P_{16}$, then prepare another system in the same state and measure, e.g., $P_{24}, P_{25}$, and $P_{26}$, and repeat these measurements many times, until enough data has been obtained to calculate the 6 mean values in (4) and the experimental value of the Bell operator for this state. 
Then, one has to repeat the experiment with different states (e.g., a partially entangled state, a product state, and a maximally mixed state). The violation predicted by $\mathrm{QM}$ is the same for every state.

There are two requirements for these experiments to be considered legitimate state-independent tests of quantum contextuality: (a) The experimental apparatus used for measuring, e.g., $P_{14}$ must be the same when $P_{14}$ is measured together with $P_{15}$ and $P_{16}$, and when it is measured together with $P_{24}$ and $P_{34}$, and must be the same for any state. (b) Every observable must be measured in different contexts. For a more detailed discussion, see [37].

Conclusions.-We have introduced 3 experimentally testable inequalities valid for any NCHV theory and violated by any quantum state. They combine the most celebrated properties of the Bell inequalities, independence of QM and experimental testability, with state independence, the most celebrated property of the KS theorem. One of these inequalities seems particularly suitable to experimentally test the state-independent violation predicted by QM.

The connection of these inequalities to previous proofs of the KS theorem and previous state-dependent inequalities gives a new insight on the relationship between the two main theorems of impossibility of hidden variables in QM. Each of the 3 introduced state-independent inequalities is related to a proof of the KS theorem based on a parity argument. An open question is whether similar state-independent inequalities can be developed for physical systems where no proofs of the KS theorem based on a parity argument are known. Specifically, an interesting open problem is finding a state-independent inequality based only on the assumption of noncontextuality for the case $d=3$.

The author acknowledges support from projects No. P06-FQM-02243 and No. FIS2008-05596.

* Electronic address: adan@us.es

[1] J. S. Bell, Physics (Long Island City, NY) 1, 195 (1964).

[2] J. F. Clauser, M. A. Horne, A. Shimony, and R. A. Holt, Phys. Rev. Lett. 23, 880 (1969).

[3] D. M. Greenberger, M. A. Horne, and A. Zeilinger, in Bell's Theorem, Quantum Theory, and Conceptions of the Universe, edited by M. Kafatos (Kluwer Academic, Dordrecht, 1989), p. 69.

[4] Č. Brukner, M. Żukowski, J.-W. Pan, and A. Zeilinger, Phys. Rev. Lett. 92, 127901 (2004).
[5] P. Hyllus, O. Gühne, D. Bruß, and M. Lewenstein, Phys. Rev. A 72, 012321 (2005).

[6] A. Acín, N. Gisin, and L. Masanes, Phys. Rev. Lett. 97, 120405 (2006).

[7] C. Schmid, N. Kiesel, W. Laskowski, W. Wieczorek, M. Żukowski, and H. Weinfurter, Phys. Rev. Lett. 100, 200407 (2008).

[8] E. P. Specker, Dialectica 14, 239 (1960).

[9] J. S. Bell, Rev. Mod. Phys. 38, 447 (1966).

[10] S. Kochen and E. P. Specker, J. Math. Mech. 17, 59 (1967).

[11] A. Peres, Phys. Lett. A 151, 107 (1990).

[12] N. D. Mermin, Phys. Rev. Lett. 65, 3373 (1990).

[13] A. Peres, J. Phys. A 24, L175 (1991).

[14] N. D. Mermin, Rev. Mod. Phys. 65, 803 (1993).

[15] A. Peres, Quantum Theory: Concepts and Methods (Kluwer, Dordrecht, 1993).

[16] A. Cabello, J. M. Estebaranz, and G. García-Alcaine, Phys. Lett. A 212, 183 (1996).

[17] D. P. DiVincenzo and A. Peres, Phys. Rev. A 55, 4089 (1997).

[18] E. F. Galvão, Ph. D. thesis, Oxford University, 2002.

[19] K. Nagata, Phys. Rev. A 72, 012325 (2005).

[20] N. Aharon and L. Vaidman, Phys. Rev. A 77, 052310 (2008).

[21] P. Heywood and M. L. G. Redhead, Found. Phys. 13, 481 (1983).

[22] A. Cabello, Phys. Rev. Lett. 86, 1911 (2001).

[23] A. Cabello, Phys. Rev. Lett. 87, 010403 (2001).

[24] A. Einstein, B. Podolsky, and N. Rosen, Phys. Rev. 47, 777 (1935).

[25] N. D. Mermin, Phys. Rev. Lett. 65, 1838 (1990).

[26] A. Cabello and G. García-Alcaine, Phys. Rev. Lett. 80, 1797 (1998).

[27] D. A. Meyer, Phys. Rev. Lett. 83, 3751 (1999).

[28] A. Kent, Phys. Rev. Lett. 83, 3755 (1999).

[29] R. Clifton and A. Kent, Proc. R. Soc. London, Ser. A 456, 2101 (2000).

[30] C. Simon, M. Żukowski, H. Weinfurter, and A. Zeilinger, Phys. Rev. Lett. 85, 1783 (2000).

[31] H. Havlicek, G. Krenn, J. Summhammer, and K. Svozil, J. Phys. A 34, 3071 (2001).

[32] D. M. Appleby, Phys. Rev. A 65, 022105 (2002).

[33] C. Simon, Č. Brukner, and A. Zeilinger, Phys. Rev. Lett. 86, 4427 (2001).

[34] J.-Å. Larsson, Europhys. Lett. 58, 799 (2002).

[35] A. Cabello, Phys. Rev. A 65, 052101 (2002).

[36] Y.-F. Huang, C.-F. Li, Y.-S. Zhang, J.-W. Pan, and G.C. Guo, Phys. Rev. Lett. 90, 250401 (2003).

[37] A. Cabello, S. Filipp, H. Rauch, and Y. Hasegawa, Phys. Rev. Lett. 100, 130404 (2008).

[38] A. A. Klyachko, M. A. Can, S. Binicioğlu, and A. S. Shumovsky, Phys. Rev. Lett. 101, 020403 (2008).

[39] Y. Nambu, arXiv:0805.3398. 